Disclosure of Interest: None declared

DOI: 10.1136/annrheumdis-2018-eular.3407

\section{AB0067 ALLOSTERIC RECEPTOR MODULATION OF A FREE FATTY ACID RECEPTOR TURNS NATURAL AGONISTS INTO POTENT ACTIVATORS OF THE SUPEROXIDE GENERATING NEUTROPHIL NADPH-OXIDASE}

L. Björkman, J. Mårtensson, A. Holdfeldt, H. Forsman, M. Gabl, M. Sundqvist, C. Dahlgren. Rheumatology and inflammation research, University of Gothenburg, Gothenburg, Sweden

Background: Short chain fatty acids are generated in the colon by bacterial fermentation of dietary fibres and serve as natural agonists for the free fatty acid receptor 2 (FFA2R/GPR43) which belongs to the large family of G-protein coupled receptors. We have earlier shown that acetate triggers an increase in the cytosolic concentration of free $\mathrm{Ca}^{2+}$ in neutrophils without any assembly/activation of the superoxide generating NADPH-oxidase (Mol Cell Biol. 2016 Sep 26;36 (20):2583-95). Allosteric modulators bind to receptors at sites topographically distinct from the agonist/antagonist binding site and can regulate receptor functions positively or negatively.

Objectives: We undertook this study to determine whether an FFA2R selective modulator affects the neutrophil response induced by natural FFA2R agonists.

Methods: Neutrophils were collected from healthy blood donors. Release of superoxide anions generated by the assembled/activated NADPH-oxidase was recorded by sensitive isoluminol/HRP amplified chemiluminescence method. Intracellular $\mathrm{Ca}^{2+}$ transients were measured with FURA 2-AM labelled neutrophils.

Results: The allosteric modulator lacked a direct activating effect on neutrophils, but turned natural FFA2R agonists into potent activating agonists that triggered not only a transient rise in the cytosolic concentration of free $\mathrm{Ca}^{2+}$ ions but also an assembly of the NADPH-oxidase. The NADPH-oxidase activity induced by the combined effect of the allosteric modulator and the natural agonist acetate could be further increased in neutrophils treated with the pro-inflammatory cytokine TNF- $\alpha$. The receptor selectivity was demonstrated through the inhibition of the neutrophil activity by the novel FFA2R antagonist CATPB. In addition, the allosteric modulator lacked effect on neutrophil responses triggered by a novel and selective agonist for the closely related GPR84, a receptor that recognises medium chain fatty acids.

Conclusions: Allosteric modulators that positively co-operate with natural FFA2R agonists and prime neutrophils in their response to such agonists may serve as good tools for further unravelling the physiological functions of the FFA2R and its involvement in various diseases. In this study, allosteric modulation of FFA2R is introduced as a receptor selective mechanism to prime neutrophils to produce increased amounts of reactive oxygen species.

\section{REFERENCE:}

[1] Björkman L, Mårtensson J, Winther M, Gabl M, Holdfeldt A, Uhrbom M, Bylund J, Højgaard Hansen A, Pandey SK, Ulven T, Forsman H, Dahlgren C. The Neutrophil Response Induced by an Agonist for Free Fatty Acid Receptor 2 (GPR43) Is Primed by Tumor Necrosis Factor Alpha and by Receptor Uncoupling from the Cytoskeleton but Attenuated by Tissue Recruitment. Mol Cell Biol. 2016 Sep 26;36(20):2583-95. doi:10.1128/ MCB.00161-16 [Print 2016 Oct 15].

Disclosure of Interest: None declared DOI: 10.1136/annrheumdis-2018-eular.3211

\section{AB0068 100 ALARMINS S100A8 AND S100A9 MODULATE THE INFLAMMATORY MICROENVIRONMENT IN EARLY TENDINOPATHY}

L.A.N. Crowe ${ }^{1}$, M. Akbar ${ }^{1}$, K. Patommel ${ }^{1}$, S.M. Kitson ${ }^{1}$, E. Garcia Melchor ${ }^{1}$, D S. Gilchrist ${ }^{1}$, G.A. Murrell ${ }^{2}$, I.B. Mclnnes ${ }^{1}$, N.L. Millar ${ }^{1} .{ }^{1}$ Institute of Infection, Immunity and Inflamamtion, University of Glasgow, Glasgow, UK; ${ }^{2}$ Department of Orthopaedic Surgery, St. Georges Hospital Campus, University of New South Wales, Sydney, Australia

Background: Alarmins- also referred to as damage associated molecular patterns (DAMPS)- are endogenous molecules mobilised in response to tissue damage known to activate the innate immune system in the early stages of disease. The molecular mechanisms that regulate inflammatory and remodelling pathways in tendinopathy are largely unknown therefore identifying early immune effectors is essential to understanding the pathology. S100A8 and S100A9 are constitutively expressed by cells of myeloid origin; under pathological conditions they are induced in other cell types in response to environmental triggers and cellular damage.

Objectives: Based on previous investigations we sought evidence of S100A8/A9 expression in human tendinopathy and thereafter, to explore mechanisms whereby $\mathrm{S} 100$ proteins may regulate release of inflammatory mediators and matrix synthesis in human tenocytes.

Methods: Torn supraspinatus tendon (established pathology) and intact subscapularis tendon (early pathology) biopsies were collected from patients undergoing arthroscopic shoulder surgery. Control samples of healthy hamstring tendon were collected from patients undergoing hamstring tendon $\mathrm{ACL}$ reconstruction. S100A8/A9 expression was analysed at transcript and protein level using quantitative RT-PCR and immunohistochemistry, respectively. Primary human tenocytes were cultured from hamstring tendon tissue. The in vitro effect of recombinant human $\mathrm{S} 100 \mathrm{~A} 8 / \mathrm{A} 9$ on human tenocytes was measured using quantitative RT-PCR and release of inflammatory mediators was measured at protein level by ELISA

Results: Immunohistochemical staining of tendinopathic tissues indicated the presence of S100A8 and S100A9 in tendinopathy with early diseased tissue dis playing a distinct increase in S100A8 and S100A9 expression compared with control and established pathology. These findings were mirrored by data obtained at transcript level from both early and late pathology. Treating tenocytes with exogenous S100A8/9 significantly increased release of IL-6 and CCL2; however, no alterations in genes associated with matrix remodelling were observed at a transcript level.

Conclusions: The presence of S100A8 and S100A9 in early tendinopathic lesions suggests expression is upregulated in response to cellular damage. We have confirmed the presence of S100A8, S100A9, CCL2 and IL-6 in tendinopathy and propose that S100A8 and S100A9 participate in early pathology by modulating the stromal microenvironment and influencing the inflammatory profile of tenocytes. S100A8 and S100A9 may participate in a positive feedback mechanism involving enhanced leukocyte recruitment and release of pro-inflammatory cytokines from tenocytes that perpetuates the inflammatory response within the tendon in the early stages of disease. This, in turn, may contribute aberrant matrix remodelling and associated morphological deficiencies within the tendon. We pro pose S100A8 and S100A9 are active alarmins in early tendinopathy that indirectly influence matrix remodelling by perpetuating the stromal inflammatory environ ment. Selective targeting of DAMP signalling may offer novel therapeutic approaches in the management of human tendon disorders.

\section{REFERENCE:}

[1] Millar NL, et al. MicroRNA29a regulates IL-33-mediated tissue remodelling in tendon disease. Nat Commun 2015;6:6774.

Disclosure of Interest: None declared

DOI: 10.1136/annrheumdis-2018-eular.7019

\section{AB0069 INCREASED EXPRESSION OF SOLUBLE MIC-A IN THE SYNOVIAL FLUID OF RHEUMATOID ARTHRITIS} PATIENTS

L. Bernardi ${ }^{1,2}$, A. Mariotte ${ }^{1}$, A. Scanu ${ }^{2}$, D. Noël ${ }^{3}$, L. Punzi ${ }^{2}$, S. Bahram ${ }^{1}$ P. Georgel ${ }^{1}$, J. Sibilia ${ }^{1}$. 'INSERM UMR_S 1109, Fédération de Médecine Translationnelle de Strasbourg, Université de Strasbourg, Strasbourg, France; ${ }^{2}$ Rheumatology Unit, Department of Medicine-DIMED, University of Padova, Padova, Italy, ${ }^{3}$ INSERM UMR S 1183, Montpellier University, Montpellier, France

Background: MIC-A (Major histocompatibility complex class I chain-related gene $A)^{1}$ is a transmembrane or soluble protein that interacts with the activating NKG2D receptor. MIC-A stimulates effector responses mediated by NK and CD8 + T cells under cellular stress conditions, like cancer or infections. ${ }^{2}$ MIC-A is also associated with autoimmune diseases (such as rheumatic disorders) character ised by immune dysregulation triggered by environmental factors, and plays important roles in immune activation and surveillance. ${ }^{3}$ In mice, various NKG2D ligands were discovered: Rae-1, $\mathrm{H} 60$ and MULT1 families. ${ }^{4}$

Objectives: This study aims at investigating the potential pathological relevance of soluble MIC-A (sMIC-A) protein in inflammatory rheumatic diseases involving articular structures in humans. The expression of orthologous NKG2D ligands in mouse models of experimental joint inflammation is also quantified.

Methods: We collected synovial fluid (SF) from 118 subjects: 22 Rheumatoid Arthritis (RA), 13 Psoriatic Arthritis (PSOA), 12 Gout Disease (GOUT), 18 Calcium 
Pyrophosphate Deposition Disease (CPPD), 8 Reactive Arthritis (REA) and 45 Osteoarthritis patients. Gout and CPPD diseases were confirmed by the presence of crystals in SF. Clinical data were collected. The concentration of soluble MIC-A (sMIC-A), interleukin (IL)-1, IL-6 and IL-8 was measured by ELISA. Murine Rae1, H60 and Mult1 transcripts were quantified by real-time quantitative PCR (RTqPCR) in 3 models of joint inflammation: Serum Transfer Arthritis (STA), Collagen-induced arthritis $(\mathrm{CIA})$ and Collagenase-Induced Osteoarthritis $(\mathrm{CIOA})$. Results: Significantly higher levels of sMIC-A were found in the synovial fluid of RA patients in comparison with all others diseases ( $<<0.001$, figure n.1). sMIC-A levels were correlated to white blood cell counts and levels of inflammatory cytokines IL-1, IL-6 and IL-8. Similarly, higher expression levels of Rae-1, H60 and Mult1 were found in chronic arthritis mouse models in comparison with osteoarthritic mice.

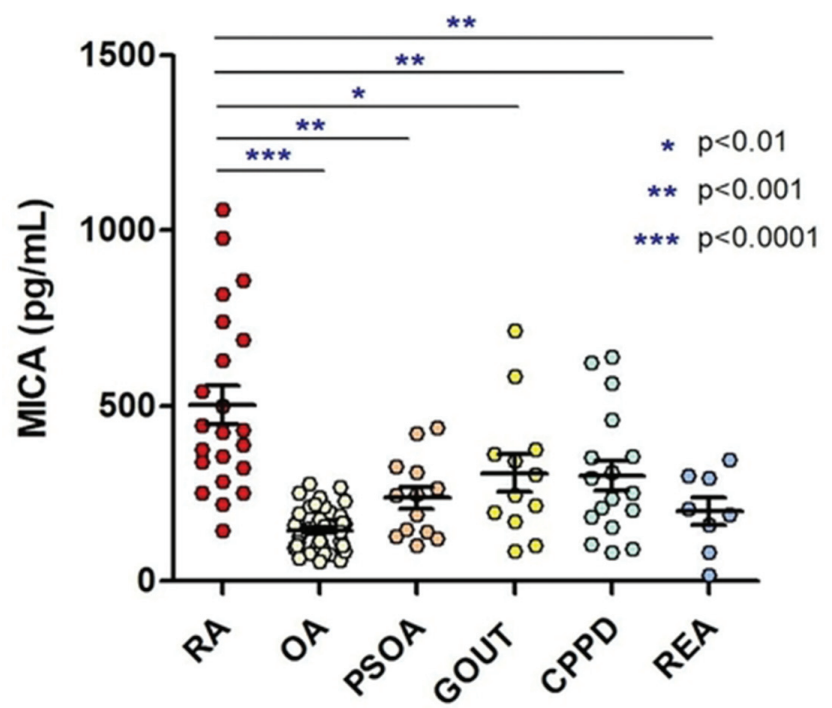

Abstract AB0069 - Figure 1

Conclusions: Our data identifies synovial SMIC-A as an important player in rheumatoid arthritis compared to other rheumatic diseases and osteoarthritis. Investigations in mouse models are in agreement with this finding

\section{REFERENCES:}

[1] Bahram, Seiamak, et al. A second lineage of mammalian major histocompatibility complex class I genes. Proceedings of the National Academy of Sciences 1994:91(14):6259-6263.

[2] Groh, Veronika, et al. Costimulation of CD8 $\alpha \beta$ T cells by NKG2D via engagement by MIC induced on virus-infected cells. Nature immunology $2001 ; 2(3): 255$

[3] Spear, Paul, et al. NKG2D ligands as therapeutic targets. Cancer Immunity Archive 2013;13(2):8.

[4] Carapito, Raphael, Seiamak, Bahram. Genetics, genomics, and evolutionary biology of NKG2D ligands. Immunological reviews 2015;267(1):88116.

Disclosure of Interest: None declared

DOI: 10.1136/annrheumdis-2018-eular.5169

\section{AB0070 ADIPOKINES AND CYTOKINES IN THE PATHOGENESIS OF PSORIASIS AND METABOLIC DISORDERS}

M. Khobeysh ${ }^{1}$, K. Sysoev ${ }^{2}$, E. Sokolovskiy ${ }^{1}$, S. Lapin ${ }^{3} .{ }^{1}$ Department of Dermatology and Veneral Diseases, Academician I.P. Pavlov First St. Petersburg State Medical University, ${ }^{2}$ Pavlov Institute of Physiology, RAS; ${ }^{3}$ Academician I.P. Pavlov First St. Petersburg State Medical University, St. Petersburg, Russian Federation

Background: Psoriasis is a systemic immune-associated disease with a specific comorbidity. The manifestations of the metabolic syndrome in this category of patients are changes that develop on systemic immune-associated inflammatory psoriatic process background and contribute to the progression of chronic inflammation.

Objectives: The aim of the study was determination of the main adipokines and cytokines content in the serum of peripheral blood, severity and activity of the disease in patients with psoriasis; clinical and laboratory evaluation of metabolic disorders. The interconnection between the production of adipokines and cytokines in psoriasis was analysed depending on the severity and activity of the psoriatic process and the nature of metabolic disorders.

Methods: Serum levels of adipokines (C-peptide, ghrelin, insulin, glucagon, leptin, visfatin, resistin, GIP, GLP-1 and PAl-1) and cytokines (IL-1 $\beta$, IL-1ra, IL-2, IL4, IL-5, IL-6, IL-7, IL-8, IL-9, IL-10, IL- 12 (p70), IL-13, IL-15, IL-17, eotaxin, FGF-2, G-CSF, GM-CSF, IFN- $\gamma$, IP-10, MCP-1, MIP- $1 \alpha$, MIP-1 $\beta$, PDGF-BB, RANTES, TNF- $\alpha$ and VEGF) were measured in 36 patients with moderate and severe psoriasis, psoriatic arthritis. There was control group of 15 basically healthy persons. Clinical and laboratory evaluation of metabolic disorders (BMI, dyslipidemia, carbohydrate metabolism disorders) and cardiovascular diseases was performed for all examined patients. The duration, severity and the amount of body surface area involved in psoriasis were evaluated in all patients using recommended indices (BSA, PASI).

Results: Patients with psoriasis showed an increase in the production of glucagon, leptin, visfatin, GLP-1 ( $\mathrm{p}<1,0 \mathrm{E}-03)$ and a decrease in the level of $\mathrm{C}$-peptide, insulin, GIP, PAI-1, resistin ( $p<1,0 \mathrm{E}-06$ ) compared with the control group. The difference of ghrelin concentrations in both group was not statistically significant. Patients with psoriasis showed an increase in the level of IL-1 $\beta$, IL-1 ra, IL-2, IL-4, IL-5, IL-6, IL-8, IL-9, IL-10, IL-12 (p70), IL-13, IL-15, IL-17, eotaxin, FGF-2, GMCSF, IFN- $\gamma$, IP-10, MCP-1, MIP- $1 \beta$ and TNF- $\alpha$ compared to control group $(\mathrm{p}<0$, 01). Positive correlations between the level of adipokines and cytokines were revealed.

Conclusions: The obtained data allow us to define adipokines as mediators between immune and endocrine systems. The imbalance between the proinflammatory and anti-inflammatory effects of adipokines observed in psoriasis demonstrates lipid metabolism dysfunction as one of the possible provoking factors of chronic inflammation determining the severity of the underlying disease. According to the results of the study, the following biological adipokines and cytokines should be classified as early biological markers of severity of the psoriatic immune-associated inflammatory process, with all its comorbid risks: GLP-1, glucagon, leptin, wisfatin, IL-1ra, IL-2, IL-4 IL-5, IL-6, IL-7, IL-8, IL-12, IL-13, IL-17, eotaxin, FGF-2, GM-CSF, IFN- $\gamma$, IP-10, MCP-1, MIP-1 $\beta$, TNF- $\alpha$. The levels of adipokines and cytokines are probably the earliest biological markers in patients with metabolic syndrome and psoriasis, the control of adipokines and cytokines level can be used to optimise therapy.

Disclosure of Interest: None declared

DOI: 10.1136/annrheumdis-2018-eular.5684

\section{$\mathrm{AB} 0071$ \\ EFFECTS OF CHONDROITIN SULPHATE AND GLUCOSAMINE ON INFLAMMATORY CYTOKINES IN MACROPHAGES}

M.-F. Hsueh ${ }^{1}$, E. Montell ${ }^{2}$, V.B. Kraus ${ }^{1} .{ }^{1}$ Molecular Physiology, Duke University, Durham, USA; ${ }^{2}$ Pre-Clinical RandD Area, Bioibérica, S.A.U., Barcelona, Spain

Background: The combination of pathogen-associated molecular patterns (PAMPs), such as lipopolysaccharide (LPS), and endogenous danger-associated molecular patterns (DAMPs), such as hyaluronan (HA) fragments, are known to be able to induce an inflammatory response from macrophages characterised by the release of pro-inflammatory cytokines ${ }^{1}$. We have previously shown that pharmaceutical grade chondroitin sulphate (CS), commonly used in the symptomatic treatment of osteoarthritis $(\mathrm{OA})$, can attenuate the inflammatory response.

Objectives: To evaluate the synergistic effects of CS and glucosamine (GLU) in combination on LPS/HA mediated inflammatory responses of an immortalised human macrophage cell line (THP-1) and primary synovial fluid cells.

Methods: THP-1 monocyte cells were grown and differentiated into mature macrophages by the addition of $200 \mathrm{nM}$ of phorbol 12-myristate 13-acetate (PMA) as previously described ${ }^{1}$. Cells were cultured with a physiologically achievable range of concentrations of CS and GLU $(0,10,50,200 \mu \mathrm{g} / \mathrm{ml}$ of each, Bioibérica, S.A. U.) for 6 hours, then primed with physiologically relevant concentrations of LPS $(10 \mathrm{ng} / \mathrm{ml})$ ( $\mathrm{n}=12 /$ group). After 24 hours, cell culture media were replaced with serum free Opti-MEM supplemented with the previously mentioned concentrations of CS and GLU, LPS, and $10 \mu \mathrm{g} / \mathrm{ml}$ HA fragments (ultra-low molecular weight, Lifecore). After a further 24 hours, supernatants were harvested for NF-K $\mathrm{B}$ activity and pro-inflammatory cytokine (IL-1 $1 \beta, \mathrm{IL}-6, \mathrm{IFN}-\gamma$, and TNF- $\alpha$ ) assessment. Cell viability was measured using PrestoBlue reagent. The human knee 